EUROPEAN ORGANIZATION FOR NUCLEAR RESEARCH

CERN - SL DIVISION

CERN-SL-2000-056 BI

\title{
SIGNAL PROCESSING FOR BEAM POSITION MONITORS
}

\author{
Vismara G.
}

\begin{abstract}
At the first sight the problem to determine the beam position from the ratio of the induced charges of the opposite electrodes of a beam monitor seems trivial, but up to now no unique solution has been found that fits the various demands of all particle accelerators. The purpose of this paper is to help "instrumentalists" to choose the best processing system for their particular application, depending on the machine size, the input dynamic range, the required resolution and the acquisition speed. After a general introduction and an analysis of the electrical signals to be treated (frequency and time domain), the definition of the electronic specifications will be reviewed. The tutorial will present the different families in which the processing systems can be grouped. A general description of the operating principles with relative advantages and disadvantages for the most employed processing systems is presented. Special emphasis will be put on recent technological developments based on telecommunication circuitry. In conclusion, an application example will show how to choose the correct solution for a particular case
\end{abstract}




\title{
Signals processing for Beam Position Monitors
}

\author{
Giuseppe Vismara
}

CERN - Geneva-23 - Switzerland

\begin{abstract}
At the first sight the problem to determine the beam position from the ratio of the induced charges of the opposite electrodes of a beam monitor seems trivial, but up to now no unique solution has been found that fits the various demands of all particle accelerators. The purpose of this paper is to help "instrumentalists" to choose the best processing system for their particular application, depending on the machine size, the input dynamic range, the required resolution and the acquisition speed. After a general introduction and an analysis of the electrical signals to be treated (frequency and time domain), the definition of the electronic specifications will be reviewed. The tutorial will present the different families in which the processing systems can be grouped. A general description of the operating principles with relative advantages and disadvantages for the most employed processing systems is presented. Special emphasis will be put on recent technological developments based on telecommunication circuitry. In conclusion, an application example will show how to choose the correct solution for a particular case.
\end{abstract}

\section{INTRODUCTION}

The beam position monitor (BPM) can be found in every accelerator and is by far the most popular instrument, particularly useful for machine setting-up and developments.

The BPM has largely evolved since the early days, from the simple scope visualization of coaxial multiplexed P.U. signals into a very complex system. They are now capable of digitizing individual bunches separated by a few tenths of ns, with spatial resolution in the micron range, while the resulting orbit or trajectory collected from several hundred PUs can be displayed in a fraction of a second.

To obtain such a performance the processing electronics have to be optimized to the machine and beam parameters.

A unique solution capable of covering all the possible combinations with satisfactory results seems almost impossible to realize. This is the reason for the wide spectrum of signal processing in use today.

The BPM applications are not only limited to orbit and trajectory measurements, they can also perform static and dynamic beam parameter measurements by exploiting the large amount of data collected and stored in their memories ${ }^{1}$.

By a "turn-by-turn" acquisition on a single monitor, it is possible to measure betatron oscillations, beam response and transfer function.

By a "turn-by-turn" acquisition using the whole monitoring system, it is possible to measure phase advance, local chromaticity, phase change versus beam current due to transverse reactive impedance and to perform optics checks.

By averaging over 1000 or more revolutions, high-resolution measurements can be obtained. The orbit response to local deflections is used for beam optics computing while machine impedance measurements are possible from the current dependence of the orbit. 
A very recent application is energy calibration. In the LEP machine stable, polarized beam at $45 \mathrm{GeV}$ is used to calibrate a spectrometer, which can then at $>100$ $\mathrm{GeV}$ measure the energy within a resolution of $10^{-4}$. Three $\mathrm{X}$ and $\mathrm{Y}$ monitors on each side of the deflecting magnet, with very high resolution $(<1 \mu \mathrm{m})$ and long term and temperature stability, are required.

The BPM is also used for feedback applications to stabilize and align the beam; in this case, speed (loop bandwidth) is more important than linearity.

Another application (ESRF \& SPS) for the BPM is the beam position interlock ${ }^{2}$ which dump the beam when an excessive displacement is detected for a certain time.

These applications are much more performance demanding than for a simple position measurement. They require an excellent linearity over a large part of the chamber aperture, a high-resolution $(\mu \mathrm{m})$ to avoid large beam excitation and good position stability versus beam intensity, time and temperature variation.

\section{SIGNAL ANALYSIS}

\subsection{Signal Source}

The reference parameter used to define the input signal in a processing system is the beam current circulating in the machine. The beam current depends on the injected charge and the revolution period. The relation between current and charge is:

$$
\mathrm{I}_{\mathrm{b}}=\mathrm{Q}_{\mathrm{b}} * \mathrm{~N}_{\mathrm{b}} / \mathrm{t}_{\mathrm{rev}}
$$

Where $\mathrm{Q}_{\mathrm{b}}$ is the charge per bunch in Coulombs, $\mathrm{N}_{\mathrm{b}}$ is the number of bunches and $\mathrm{t}_{\mathrm{rev}}$ is the revolution period.

The beam current is the correct parameter when defining the sensitivity of the processing system if it will integrate over one or more revolution cycles. When using wide-band processing systems, capable of resolving individual bunches, it is much more convenient to talk about charges per bunch.

Another parameter defining the input signal is the longitudinal charge density of a bunch. It can be assumed to be almost gaussian for leptons and cosine-square for protons, hence the induced signals at the PU's output will have almost the same shape. The bunch length depends on the voltage gradient and the beam energy.

It is also assumed that the transverse beam size is negligible relative to the PU aperture but for $\mu \mathrm{m}$ resolution it must be taken into account ${ }^{3}$.

In order to translate the $\mathrm{I}_{\mathrm{b}}$ or $\mathrm{Q}_{\mathrm{b}}$, into the electrical voltage seen by the signal processing system, it is very convenient to define, in analogy to Ohm's law, the following relation:

$$
\mathrm{V}(\mathrm{t})=\mathrm{Z}_{\mathrm{t}} * \mathrm{I}_{\mathrm{b}}(\mathrm{t})
$$

Where $\mathrm{Z}_{\mathrm{t}}$ is called the transfer impedance of the PU.

\subsection{Beam Structure}

Particle accelerators can be filled with beam in various ways; these determine the signal spectral content that the processing electronics can exploit. 
It is possible to define 4 major beam structures.

All rf buckets filled: Beam is trapped by the rf cavities in such a way that a particle bunch is present in all $\mathrm{rf}$ buckets. This situation is the easiest to be treated since the induced signal presents an almost monochromatic frequency spectrum. This is a typical condition for machines optimized for the maximum beam current (e.g. PEP II, LEP fixed target operation).

Few rf buckets filled: (with longitudinal symmetry): Beam bunches are only present in a regular structure over $\mathrm{N}$ possible buckets. The bunch density is the highest possible. The harmonics content shows the revolution frequency and all the multiple frequencies of [1 / bunch spacing] (e.g. LEP).

Variable buckets filled: In some particular cases the machine is filled with a particular structure, which is not longitudinally symmetric. A large spectral harmonic content is present (e.g. LHC).

Un-bunched beam: This situation can only be present in the absence of an rf signal, exclusively for proton or heavy ions machines (e.g. Cebaf recirculating linac ). This is the most difficult structure to process, since an unstructured beam is almost impossible to treat by the processing systems discussed here.

Some authors summarize the above beam filling structures by introducing the notion of a beam-bunching factor. It is defined as the ratio between the bunch width and the bunch spacing.

\subsection{Beam Position Monitors}

Five PU families are nowadays employed: Electrostatic (Buttons, electrodes of various shapes,..), Electromagnetic (Couplers), Resonant cavity4 (specially suited for H.F. linacs), Magnetic and Resistive (wall current).

I will briefly describe the most commonly used.

\subsubsection{Electrostatic (Capacitive)}

Let us analyze the properties of buttons since they are the easiest and cheapest to build. The image current associated with a circulating beam will induce a charge directly proportional to the beam intensity and inversely proportional to the distance.

Their simplified equivalent circuit is the following:

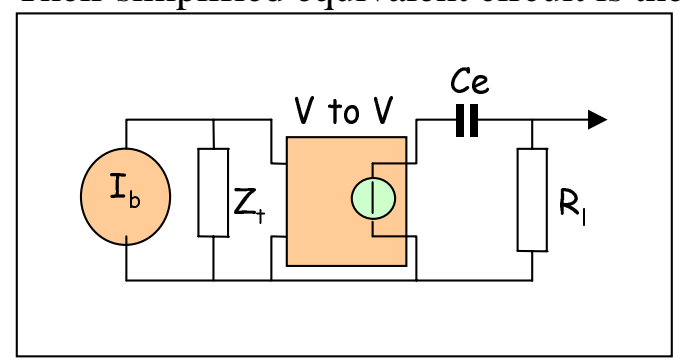

FIGURE 1. Simplified button's equivalent circuit

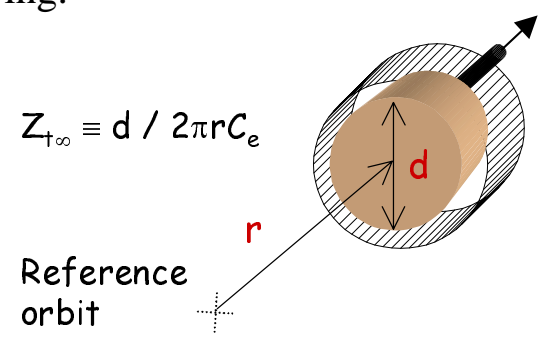

FIGURE 2. Button transfer impedance

where $Z_{t}$ is the transfer impedance as defined above, $C_{e}$ is the electrode capacitance and $\mathrm{R}_{1}$ is the load impedance.

The capacitive coupling is responsible for the low frequency cut-off, which depends on the PU time constant $\mathrm{T}=\mathrm{R}_{\mathrm{l}} \mathrm{C}_{\mathrm{e}}$. Its value is in the hundreds of $\mathrm{MHz}$ range. 
When designing button PUs special care should be taken to suppress unwanted parasitic signals due to the following phenomena:

- The button geometry determines a resonant cavity between the button and the body of the vacuum pipe; this resonator is usually tuned to $\mathrm{GHz}$ frequencies and can be excited by short bunches.

- The impedance variation on the transmission line from vacuum to air (ceramic or glass dielectric) will produce impedance mismatching, hence reflections, generating standing waves.

To attenuate these unwanted signals most processing systems have a front-end lowpass filter; in addition, the interconnection cables play a similar role as LP filters. The resulting global frequency response looks like a wide band-pass filter.

\subsubsection{Electromagnetic (Strip Line Couplers)}

Contrary to the electrostatic PU, here it is the electromagnetic field of the beam that induces a signal on the strip line:

$$
\mathrm{Z}_{\mathrm{t} \infty}=60 \ln [(\mathrm{r}+\mathrm{h}) / \mathrm{r}] \equiv \mathrm{Z}_{0}[\mathrm{a} / 2 \pi(\mathrm{r}+\mathrm{h})]
$$

where $\mathrm{Z}_{0}$ is the characteristic impedance and $\mathrm{a}, \mathrm{r}$, $\mathrm{h}$ are the mechanical dimensions.

The amount of obtainable signal depends on the azimuthal coverage and on the strip-line length if it is comparable to the bunch length $(2 \sigma)$.

The signal response versus frequency has a sinusoidal distribution and its maximum is obtained for a frequency corresponding to $f_{0}=1 / 4 * t$, where $t$ is the electrical coupler length. On the other hand, for a frequency corresponding to $f_{0}=1 / 2 * t$ the induced signal will be a minimum. This distribution repeats for all multiples of the fundamental base-band frequency.

Each strip-line has two termination ports and the designer can choose the output signal polarity on the downstream port by putting either a short circuit or an open end on the upstream port. When the upstream port is loaded with its characteristic impedance, no signal will appear on the downstream port, if the transverse electro-magnetic (TEM) wave and the beam speed are identical. In this case, the coupler is directional and it is possible to separate the signals of two beams circulating in opposite directions.

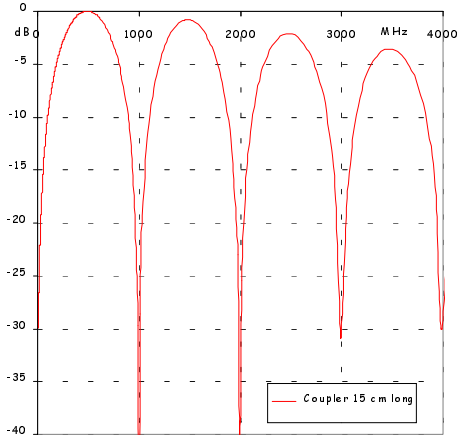

FIGURE 3. Coupler's frequency response

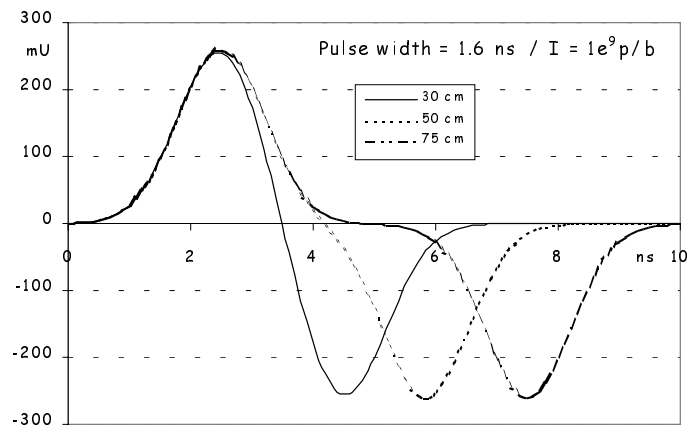

FIGURE 4. Coupler's time response 


\subsection{Beam position computing}

The beam position is reconstructed from the signal difference of opposite PUs. The induced signals are intensity and position sensitive, hence the position can only be obtained by suppressing the intensity dependence; in other words, the signals should be normalized to the beam intensity:

$$
\text { Position }=\mathrm{K}_{\mathrm{x}, \mathrm{y}} * \Delta / \Sigma=\mathrm{K}_{\mathrm{x}, \mathrm{y}} * \mathrm{~N}_{\mathrm{p}}
$$

where $\mathrm{K}_{\mathrm{x}, \mathrm{y}}$ is a scaling coefficient, $\Delta$ the electrodes signal difference and $\Sigma$ the beam intensity. For a BPM having a linear transfer function and the electrodes placed on a circular surface (of the vacuum pipe), $\mathrm{K}_{\mathrm{x}, \mathrm{y}}$ corresponds to its radius (Figure 5).

\subsubsection{Normalized Position ( $\mathrm{Np}$ )}

In the above equation, $\mathrm{N}_{\mathrm{p}}$ represents the normalized position. It is dimensionless and can vary from -1 unit $(\mathrm{U})$ to $+1 \mathrm{U}$ passing through 0 for a centered beam.

$1 \mathrm{U}$ is called the "Normalized half aperture" $\left(\mathrm{N}_{\mathrm{a}}\right)$. All parameters defining the performance of an analog signal processing system should be specified relative to $\mathrm{N}_{\mathrm{a}}$.

Button or coupler monitors have strongly nonlinear transfer functions, with higher sensitivity at the center. In this case the effective $\mathrm{K}_{\mathrm{x}, \mathrm{y}}$ corresponds to the derivative at the center of the transfer function.

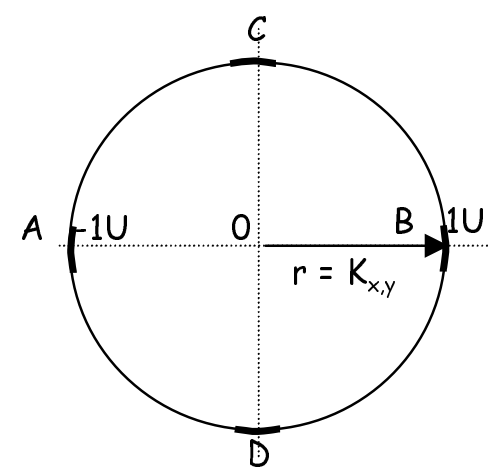

FIGURE 5. 4 PU's normalized representation

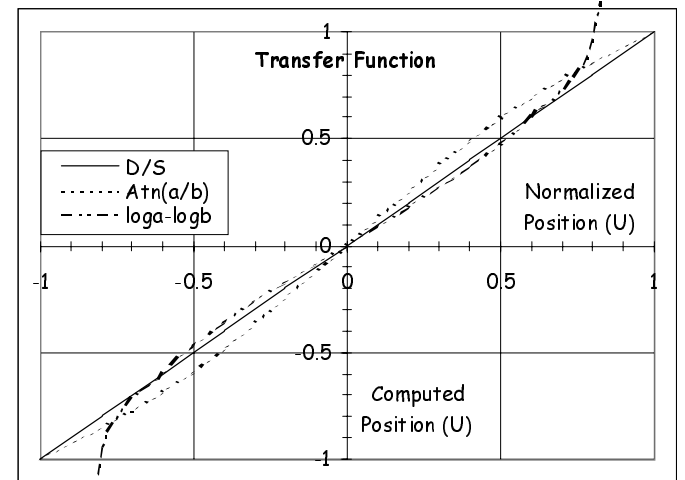

FIGURE 6. Normalized transfer functions

There are at least three methods to obtain a normalized signal.

\subsubsection{Difference over Sum}

As was explained above

$$
\mathrm{N}_{\mathrm{p}}=(\mathrm{A}-\mathrm{B}) /(\mathrm{A}+\mathrm{B})
$$

where $\mathrm{A}, \mathrm{B}$ are the induced voltage signals.

The difference and the sum can be obtained either in an analog way by the use of a $0^{\circ} / 180^{\circ}$ passive hybrid, a differential amplifier, or simply computed by software.

\subsubsection{Amplitude to phase conversion (AM/PM)}

The two input signals are converted, by the use of a $90^{\circ}$ passive hybrid, into two signals having identical amplitude but different phase. Their phase relation is

$$
\mathrm{N}_{\mathrm{p}}=\phi=2 * \text { Arctangent }(\mathrm{A} / \mathrm{B})
$$


The transfer function is not linear, and shows an S shape (Figure 6).

The slope at the center is higher, hence a large sensitivity or better $\mathrm{S} / \mathrm{N}$.

\subsubsection{Logarithmic ratio}

The two input signals are converted into their logarithmic equivalents and subtracted from each other, which is equivalent to the logarithm of their ratio.

This is a pure active analog process.

$$
\mathrm{N}_{\mathrm{p}}=[\log (\mathrm{A})-\log (\mathrm{B})]=\log (\mathrm{A} / \mathrm{B})
$$

The transfer function is non-linear, and shows a reversed $\mathrm{S}$ shape (Figure 6).

There is a very large deviation when exceeding $>70 \%$ of the $\mathrm{N}_{\mathrm{a}}$ and the sensitivity around the center is $\sim 1.5$ times smaller compared to the AM/PM function.

\section{DESIGN PARAMETER SPECIFICATIONS AND THEIR DEFINITION}

When starting the design of analog signal processing electronics, one should first define all the parameter requirements indispensable to qualify the performance of the system.

Since engineers have adopted slightly different definitions according to the function of their systems, it is quite difficult to compare the performances of the different solutions.

In this chapter, I will try to define the way each fundamental parameter should be measured.

\subsection{Accuracy}

Accuracy is the ability to determine the absolute beam position.

This is limited by mechanical tolerances and alignment, the magnetic field distribution and the electronic errors. Beam based calibration techniques allow the global offset of a processing system to be determined for a particular condition, and so establish a reference point.

From here onwards it is the processing electronics that is responsible for the absolute position. The error sources are of various origin: the parasitic element on the PU itself (spurious resonances, impedance mismatching), interconnecting cables impedance mismatching and attenuation, electromagnetic interference and input stage noise, non-linearity and intensity dependence, gain difference between channels, calibration errors, and granularity errors on the digitizer.

\subsection{Resolution}

Resolution is the minimum position difference that can be resolved.

This parameter is particularly important in colliding machines, where luminosity depends on how accurately beams can be aligned.

Two resolutions can be defined: 


\subsubsection{Single shot resolution}

This corresponds to the standard deviation (Stdev) of each individual position measurement. It applies to single beam passage (trajectory or transfer line) measurements.

\subsubsection{Absolute resolution}

This has the same definition as above but is obtained from the average over a certain time (e.g. $1 \mathrm{~ms}$ ) when digitizing at the maximum acquisition rate. It applies to circulating or repetitive beam.

The measurements should be performed under conditions corresponding to a centered beam, at its nominal intensity.

The ADC resolution should be equal to or just below the rms. value of the output noise, measured within the input bandwidth of the digitizer.

At low input level, the absolute resolution depends on the input noise and the bandwidth of the analog processor. For large input signals, the limiting factor is mainly the ADC resolution (number of bits) and the uncertainty on electronic parameters such as the time jitter.

\subsection{Stability}

Stability is one of the most important parameters in a BPM since the main purpose of the system is to give a stable reference of the center. The uncertainty on the measurement stability will affect the global resolution of system.

The position measurement should be independent of the beam intensity, the bunch shape and the bucket filling.

Stability versus input signal: It is calculated as the Stdev from a series of digitized positions, measured over the whole dynamic range.

Reproducible intensity dependent errors can be compensated by the use of a polynomial fit when measuring the beam intensity.

The measurements should be averaged, in order to reduce the noise contribution to well below the error itself and are usually recorded for a centered position.

In addition, the position measurement should be stable with respect to the time and temperature at least during the time interval between two calibrations. This is extremely important for electron machines or in applications like energy calibration.

Long term position stability (LTPS): It is defined as the Stdev of the digitized values.

Position temperature coefficient: It is the slope of the position drift versus temperature.

The measurements should be done at the nominal input level, with all other parameters being fixed. The time interval for measuring the LTPS should range from at least one hour up to few days depending on the machine, with the temperature ranging from 0 to $40 \mathrm{C}$. 


\subsection{Non-conformity to the theoretical transfer function}

This represents the measurement error relative to the theoretical transfer function.

Measurements on individual BPMs, high precision measurements (e.g. LEP spectrometer) and preservation of lepton beam spin polarization require processing systems with very low non-conformity.

Non-conformity is defined as the Stdev of the difference between the measured and the theoretical transfer function relative to $2 \mathrm{~N}_{\mathrm{a}}( \pm 1 \mathrm{U})$. The reason for this choice is determined by the fact that it corresponds to the maximum possible excursion, which in turns equals the full scale of the ADC, in all the normalization process.

The measurement should be done at the nominal input level and for constant sum signals. Since the beam excursion never reaches the extremities, the measurement can in practice be limited to $80 \%$ of the full excursion.

\subsection{Sensitivity}

Sensitivity is the minimum input level at which beam position measurement still fulfills the accuracy specifications.

In order to be able to compare among different machines, the most adequate figure is the number of charges per bunch.

The present limit for standard orbit systems corresponds to a few $10^{7}$ charges/bunch

\subsection{Dynamic Range}

The dynamic range determines the capability of the system to handle very different beam intensity conditions. Beam position measurements have to be possible, with reasonable accuracy, over the whole dynamic range.

During machine studies, circular machines are often filled with bunches of $1 \%$ o (-60 $\mathrm{dB}$ ) of the nominal intensity. The same is true for specific physics productions (e.g. Totem for LHC), and at the CERN anti-proton decelerating machine, where bunch intensity is reduced by $80 \mathrm{~dB}$ during the deceleration cycle. At GSI/Darmstadt bunch intensities can vary over several decades among proton and various ion beams.

The dynamic range is defined as the difference, expressed in $\mathrm{dB}$, between the maximum input level before a large non-linearity on the output signal appears (saturation) and the minimum input level at which a pre-defined signal to noise ratio $(\mathrm{S} / \mathrm{N})$ is reached.

A practical figure to define when saturation is reached is a $1 \mathrm{~dB}$ signal compression (= $10 \%$ error in the position measurement) while a $20 \mathrm{~dB} \mathrm{~S} / \mathrm{N}$ ratio could define the minimum input level.

Processors using a discrimination level will not be limited by the $\mathrm{S} / \mathrm{N}$ ratio, the lower limit being determined by the discriminator's threshold.

\subsection{Acquisition Time}

This parameter defines the time required for the signal processor to store a full set of data into memory. 
The importance of this parameter is related to the capability of resolving individual bunches and the absolute resolution of the processor, since the faster the system the larger the number of samples per time unit.

Some processor systems have the capability of digitizing individual bunches, others have turn by turn capability and finally some can only measure the integrated signal over several turns.

Several elements contribute to the acquisition time:

* The low-pass and band-pass filters selecting the useful frequency spectrum required for the signal processor.

* The switching and acquisition time of all channels (multiplexed signal treatment).

* The PLL time required to synchronize (synchronous detector).

* The set-up time for the automatic gain control AGC to stabilize (constant sum signal).

* The sample and hold circuit and the conversion time of the ADC.

\section{PROCESSING SYSTEM FAMILIES}

The various signal-processing systems can be grouped into different families according to the employed techniques. At least three different criteria can be used to group them.

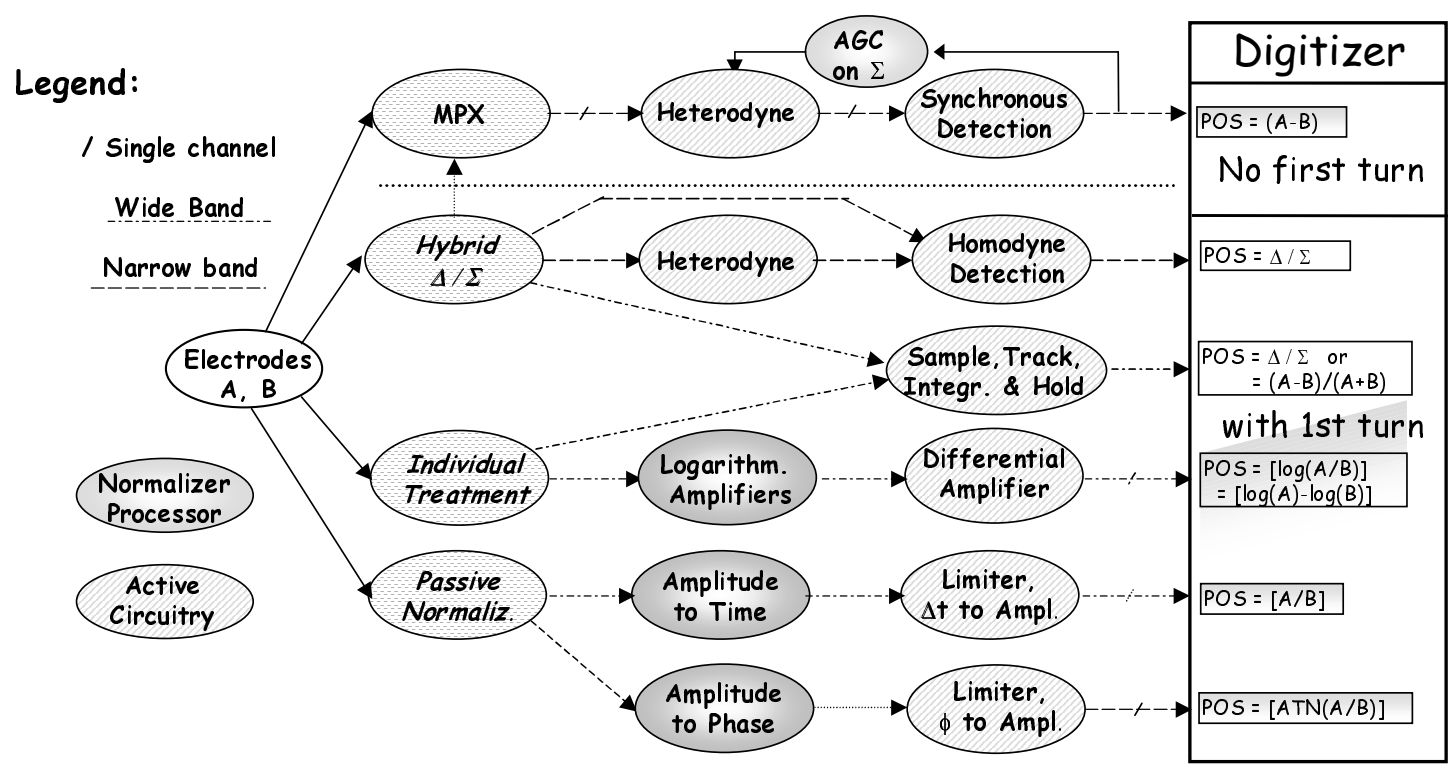

FIGURE 7. Schematic representation of the different families and their interconnections 


\subsection{Signal Recombination}

Four main categories are nowadays employed:

- Individual signal treatment: The maximum signal information is still available, therefore a wide-band processing is the most suitable. Due to a very large GainBandwidth, it offers a limited dynamic range.

- Time MPX: Electrode signals are sequentially time-multiplexed and processed by a single electronic system. It offers excellent long-term stability but cannot perform turn-by-turn measurements.

- $\underline{\Delta / \Sigma}$ : The individual signals are immediately converted by the use of hybrids into $\Delta$ and $\Sigma$. This offers excellent center position stability but requires switchable gain amplifiers.

- Passive Normalization: The signal's amplitude ratio is convert into a phase or time difference. This is amplitude independent but the intensity information is lost.

\subsection{Normalization Processes}

Normalization is an analog process that will produce a signal proportional to the position information and independent of the input signal level.

Three conditions apply to all normalization processes: 1) The intensity information is lost. 2) The digitization requires a smaller number of bits. 3) No gain selection is required.

Two active and two passive techniques are actually employed.

- Constant Sum: The Normalization is obtained by keeping constant the sum of the two electrode signals using AGC amplifiers. This approach applies to the time MPX process, where the signals exploit the same amplification chain and the digital receiver, where a permanent calibration on all channels will guarantee identical gains.

- Logarithmic Conversion: Since the ratio of the logarithm of two signals is equal to the difference of the logarithm, the signals can be converted by logarithmic amplifiers to give the normalized signal as the difference of the outputs. It offers a large dynamic range, but limited linearity.

- Amplitude to Time: This is based on the sum of a direct and a delayed signal coming from the two electrodes. The zero crossing of the sum signal varies with time proportionally to the signal ratio, and hence to the position. It offers large bandwidth but is limited to bunched beams.

- Amplitude to Phase: Is a similar process where time is replaced by phase and a single period by multiple oscillations. It is a simple solution but requires accurate phase matching (Filters).

All other processes that require computing software to extract the position information from the recorded data are known as un-normalized processes. 


\subsection{Acquisition Time}

This is the time required for the BPM to supply a full set of data to the digital processor. Three categories can be created:

- Wide-band: It groups all processing systems capable of measuring individual bunches separated by $>10$ ns down to a single bunch. The bandwidth can be as high as $100 \mathrm{MHz}$. Systems that belong to this group include: Sample/Track \& Hold, Logarithmic amplifiers, Amplitude to Time normalizers.

- Narrow-band: It groups all processing systems capable of resolving one machine revolution period and in some cases can measure individual bunches separated by $>100 \mathrm{~ns}$. The bandwidth ranges from a few $100 \mathrm{kHz}$ up to a few MHz. The Heterodyne and Amplitude to Phase processors belong to this group.

- Slow acquisition: A special class is reserved for the Time MPX processing which, while having an equivalent bandwidth relative to other heterodyne systems, is penalized in the acquisition rate by the time multiplexing.

\section{PROCESSING SYSTEM DESCRIPTIONS}

\subsection{Time Multiplexed Processor ${ }^{5}$}

The processor is conceived for closed orbit measurement of stable stored beams.

The input MPX is usually built with a multiple configuration of Ga-As switches; the channel isolation should be $>50 \mathrm{~dB}$ for frequencies up to $1 \mathrm{GHz}$.

A band-pass filter is used to select the largest line of the signal spectrum; its selectivity is not critical.

The essential element is the pre-amplifier which should handle a very large dynamic range $(>75 \mathrm{~dB})$ and compress it by $>50 \mathrm{~dB}$. Its input admittance should be kept stable as a function of the gain to avoid a zero offset drift. The global noise figure is increased by different insertion losses and should be optimized for the largest gain.

An active mixer, making use of a frequency synthesizer to reduce the noise contribution, is used to down convert the signal to a standard intermediate frequency (IF usually $10.7 \mathrm{MHz}$ or a multiple). The IF amplifier and the demodulator are usually integrated telecom circuits. The IF bandwidth is selective enough to suppress side bands at the revolution frequency (multiple bunches) but sufficiently wide to allow for

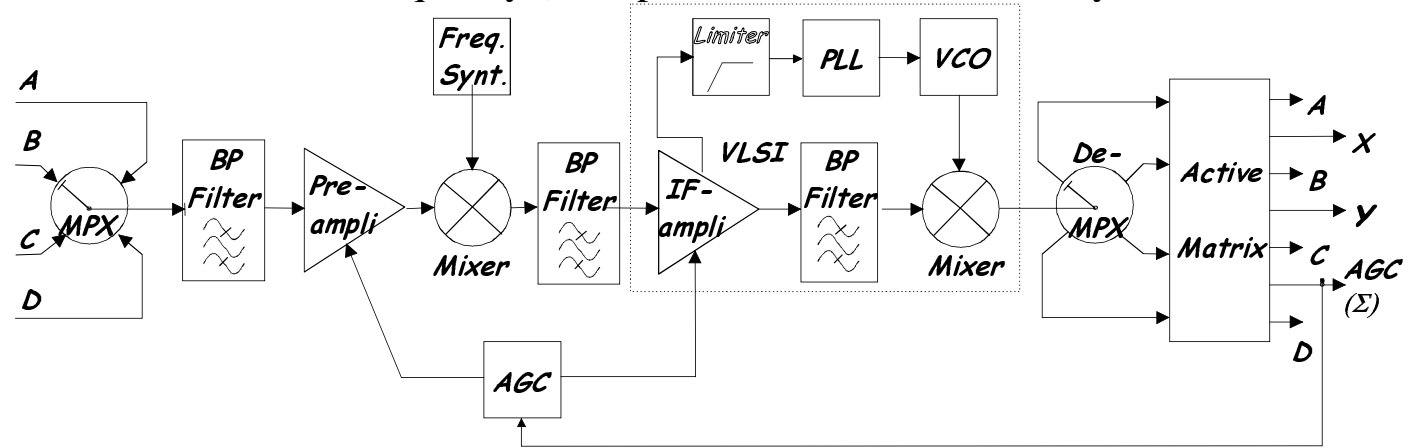

FIGURE 8. Block diagram of the time MPX processor. 
fast switching among channels $(100 \mathrm{kHz}>\mathrm{BW}<1 \mathrm{MHz})$. Synchronous detection is obtained by comparing the phase of a sample of the carrier frequency with a reference signal and driving a VCO in a phase locked loop. Synchronous detection offers a clean detected DC signal but it slows down the MPX switching time since the PLL has to relock after each switching (even with accurate phase adjustment).

The last part of the chain is composed of an output de-multiplexer, four track \& hold amplifiers and an active matrix of video amplifiers to produce the AGC sum and $\mathrm{X}, \mathrm{Y}$ positions ${ }^{6}$.

TABLE 1: Time Multiplexed processor

\begin{tabular}{|l|l|}
\hline \multicolumn{1}{|c|}{ Advantages } & \multicolumn{1}{c|}{ Limitations } \\
\hline Normalization process & Requires a stable beam during the scanning \\
\hline Reduced number of channels (x4) & No turn by turn acquisition \\
\hline Identical gain for all the channels & Slow acquisition rate (MPX) \\
\hline Large dynamic range (>80dB) & $\begin{array}{l}\text { Reduced Noise Figure (gain matching \& MPX } \\
\text { insertion losses, AGC pre-ampli.) }\end{array}$ \\
\hline Excellent position stability & $\begin{array}{l}\text { Reduced linearity, for non-linear PU's since the } \\
\Sigma \text { is not constant }\end{array}$ \\
\hline No temperature dependence and component aging & Complex engineering \\
\hline
\end{tabular}

\section{2. $\Delta / \Sigma$ Processor}

When using this approach it is convenient to convert the input signals into their equivalent difference and sum at the earliest possible stage. This is done by a simple and reliable passive element called the " $180^{\circ}$ hybrid". The input signals should be inphase, which means tight tolerances on the interconnection cables. Since the hybrid is radiation resistant, it can be connected directly to the electrodes.

Wide-band: The LP filters will just stretch the pulses. The pre-amplifiers have a large $\mathrm{BW}$ and programmable gain but a limited dynamic range. For long bunches, the $\mathrm{S} \& \mathrm{H}$ circuits are suppressed and FADCs (1 GS/s) digitize the signal directly ${ }^{7}$.

Narrow Band: In most of the cases, the hardware is similar to that of the time MPX. In some applications, the heterodyne conversion is suppressed. The pre-amplifiers have low noise figure $(\mathrm{NF}<2 \mathrm{~dB})$, programmable gain through pin diodes switches, and will absorb a large input dynamic range $(>90 \mathrm{~dB})$. A fraction of the $\Sigma$ signal is limited and used as a local oscillator in a homodyne detector. The $\Delta \& \Sigma$ signals are digitized by a track and hold circuit and externally triggered ADCs. This scheme is

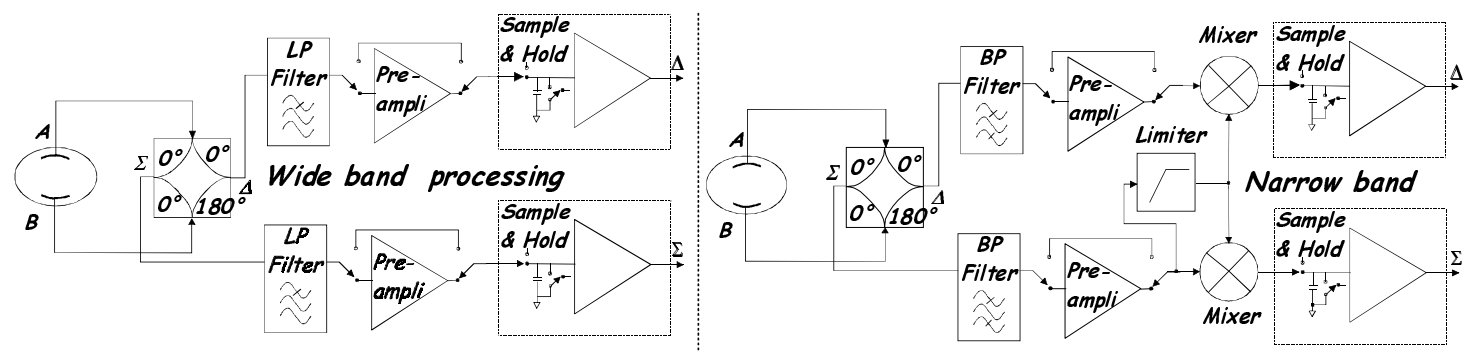

FIGURE 9. Block diagram of the $\Delta / \Sigma$ processors 
also used for single bunch measurement in complex injector machines (SPS) ${ }^{8}$, where the bunch excites the BP filter to resonate on its central frequency (see amplitude to phase normalizer). No hardware modifications are necessary but even tighter tolerances on the phase matching are required.

TABLE 2. $\Delta \& \Sigma$ Processor

\begin{tabular}{|l|l|}
\hline \multicolumn{1}{|c|}{ Advantages } & \multicolumn{1}{c|}{ Limitations } \\
\hline $\begin{array}{l}\text { The central position is independent of } \\
\text { input intensity }\end{array}$ & Programmable gain amplifiers \\
\hline Intensity measurement is available & Multiple calibration coefficients \\
\hline Excellent Noise Figure & The absolute position is $\mathrm{f}($ gain $)$ \\
\hline $\begin{array}{l}\text { [Wide band allows measurements on } \\
\text { multiple bunches }(\Delta \mathrm{t}<20 \mathrm{~ns})]\end{array}$ & $\begin{array}{l}\{\text { Tight phase matching }(\Delta, \Sigma) \text { at all the gains required } \\
\left.\text { by the synchronous detection }\left( \pm 5^{\circ}\right)\right\}\end{array}$ \\
\hline$\{$ Large dynamic range $>90 \mathrm{~dB}\}$ & $\{$ Pedestal error on $\Sigma\}$ \\
\hline
\end{tabular}

[W.B.] \& \{N.B. $\}$

\subsection{Logarithmic Amplifiers 9, 10,11}

The processor makes use of logarithmic amplifiers to compress each signal. The outputs are at first demodulated, filtered and applied to a differential amplifier.

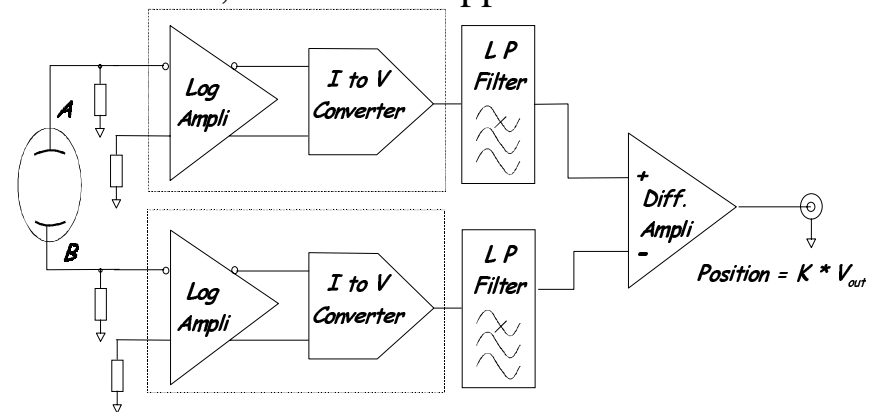

FIGURE 10. Block diagram of the logarithmic amplifier processor

The schematic is very simple but the hardware realization hides a very sophisticated electronic circuit in order to approach the ideal function. New generation circuits make use of several cascaded limiting amplifiers, with fixed gain and a wide bandwidth. Full wave rms. detectors are applied at each stage and by summing their output signals, a good approximation to a logarithmic transfer function is obtained.

TABLE 3. State of the art parameters for Logamp.

\begin{tabular}{|l|l|l|}
\hline Input dynamic range & $>90$ & $\mathrm{~dB}$ \\
\hline Input noise & $<1.5$ & $\mathrm{nV} / \sqrt{ } \mathrm{Hz}$ \\
\hline Non conformance linearity & $< \pm 0.3$ & $\mathrm{~dB}$ \\
\hline Limiter Bandwidth & D.C. to $>2$ & $\mathrm{GHz}$ \\
\hline Video Bandwidth & D.C. to 30 & $\mathrm{MHz}$ \\
\hline
\end{tabular}

The demand of the consumer market (primarily telecommunications) for these products has resulted in a wide variety of new circuits, each one optimized for a specific parameter. 
TABLE 4. Logarithmic amplifiers.

\begin{tabular}{|l|l|}
\hline \multicolumn{1}{|c|}{ Advantages } & \multicolumn{1}{c|}{ Limitations } \\
\hline $\begin{array}{l}\text { Possible applications in the time and } \\
\text { frequency domain (NB \& WB) }\end{array}$ & $\begin{array}{l}\text { State of the art performances are not } \\
\text { simultaneously available }\end{array}$ \\
\hline Very large dynamic range $(>90 \mathrm{~dB})$ & $\begin{array}{l}\text { Poor position stability vs. input level, } \\
\text { for peculiar conditions }\end{array}$ \\
\hline Wide input bandwidth & Limited linearity ( few \% of the $\left.\mathrm{N}_{\mathrm{a}}\right)$ \\
\hline No bunch shape dependency & Limited long term stability \\
\hline Simultaneous digitization of + and - charges & Temperature dependence \\
\hline Simple engineering & \\
\hline
\end{tabular}

\subsection{Amplitude to Phase Normalization}

This technique was first developed for RF signals working in the frequency domain and rapidly adapted to short pulses working in the time domain ${ }^{12}$.

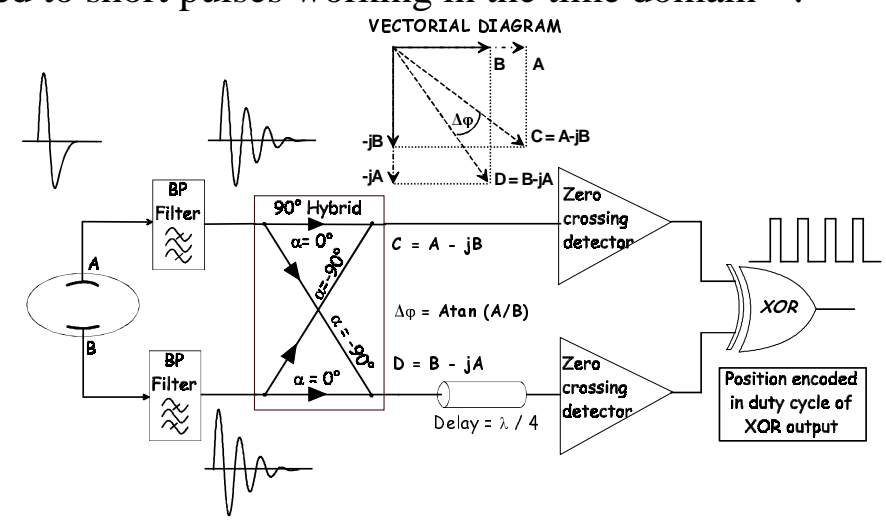

FIGURE 11. Block diagram of the amplitude to phase (AM/PM) processor

The two electrode signals are converted into a RF burst or a permanent RF signal, according to the beam shape, by the use of a BP filter. These in-phase signals are applied to the inputs of a " $90^{\circ}$ Hybrid". Each signal is split into two branches; one of them is shifted by $90^{\circ}$ and added to the opposite in-phase signal, and vice versa. The outputs are of equal amplitude and have a phase difference $(\Delta \varphi)$ proportional to the position.

$$
\text { Position } \equiv \Delta \varphi=2 * \text { Arc-tangent }(\mathrm{A} / \mathrm{B})-\pi / 2
$$

This relation is valid for both a continuous wave or for a burst after proper settling time, which depends on the bandwidth. To avoid an ambiguity in sign, one output is also delayed by $90^{\circ}$ degrees.

The two signals are applied to comparators that suppress the amplitude dependence. The phase difference is reconverted into an amplitude variation by the use of XOR logic. The position information has a variable duty cycle at twice the filter frequency, and is digitized by an ADC driven by a LP filter and a video buffer.

Two cases should be distinguished:

Current modulated beam: The BP filters are tuned to the largest line in the frequency spectrum. Their selectivity (BW) should suppress all spurious frequencies (rejection $>40 \mathrm{~dB}$ ) but be wide enough to accept the small frequency changes that may occur during an acceleration cycle. The BP filters are not critical elements and the phase shift needs only be matched to within a few percent. 
Frequency down conversion $^{13}$ : For frequencies above $150 \mathrm{MHz}$, comparators are less performant and the signal is therefore down converted using heterodyne conversion. The acquisition time is increased by the ratio $f_{\mathrm{r} f} / \mathrm{f}_{\mathrm{if}}$.

Bunch modulated beams (single or multi bunches): The induced signals charge the BP filter, which in turn will start a free oscillation on its central frequency for a predetermined time. The pulse width should be shorter than the oscillation period $\left(\mathrm{W}_{\text {(fwhm) }}<1 / 4 * \mathrm{f}_{0}\right)$ to obtain the maximum signal. The bunch spacing should be larger than the damping time of the filter to allow for individual measurements. Acquisition rates up to a few $\mathrm{MHz}$ can be achieved, so that turn-by-turn and individual bunch acquisition is feasible. Since the BP filter selectivity is not critical, a single resonator can be employed. However, this results in a longer damping time and therefore requires a longer bunch separation ${ }^{14}$. Several BP filter parameters should be accurately matched in order to preserve the relative phase over several oscillation periods.

TABLE 5. Amplitude to Phase Normalization.

\begin{tabular}{|l|l|}
\hline \multicolumn{1}{|c|}{ Advantages } & \multicolumn{1}{c|}{ Limitations } \\
\hline Normalization process & Upper frequency limit $<150 \mathrm{MHz}$ \\
\hline Reduced number of channels $(\mathrm{x} 2)$ & Tight phase adjustment \\
\hline Input dynamic range $>50 \mathrm{~dB}$ & [Minimum bunch spacing (>100 ns)] \\
\hline $\begin{array}{l}\sim 10 \mathrm{~dB} \text { reduction on the position dependent } \\
\text { dynamic due to signal recombination }\left(90^{\circ} \text { sum }\right)\end{array}$ & [Matched pair BP filters (tight tolerances)] \\
\hline $\begin{array}{l}\text { [Dynamic range is independent of the number of } \\
\text { bunches] }\end{array}$ & $\begin{array}{l}\text { Hardware; limited technological improvements } \\
\text { foreseen }\end{array}$ \\
\hline Simultaneous digitization of + and - charges & \\
\hline Simple \& Reliable & \\
\hline
\end{tabular}

\{Current modulated beams $\}$, [Bunched beams]

\subsection{Amplitude to Time Normalization}

This new normalization idea is derived from the "Amplitude to phase" principle where "phase" is replaced by "time" and the applied signal has a single oscillation period. It applies to bunched beams and works in the time domain ${ }^{15,16}$.

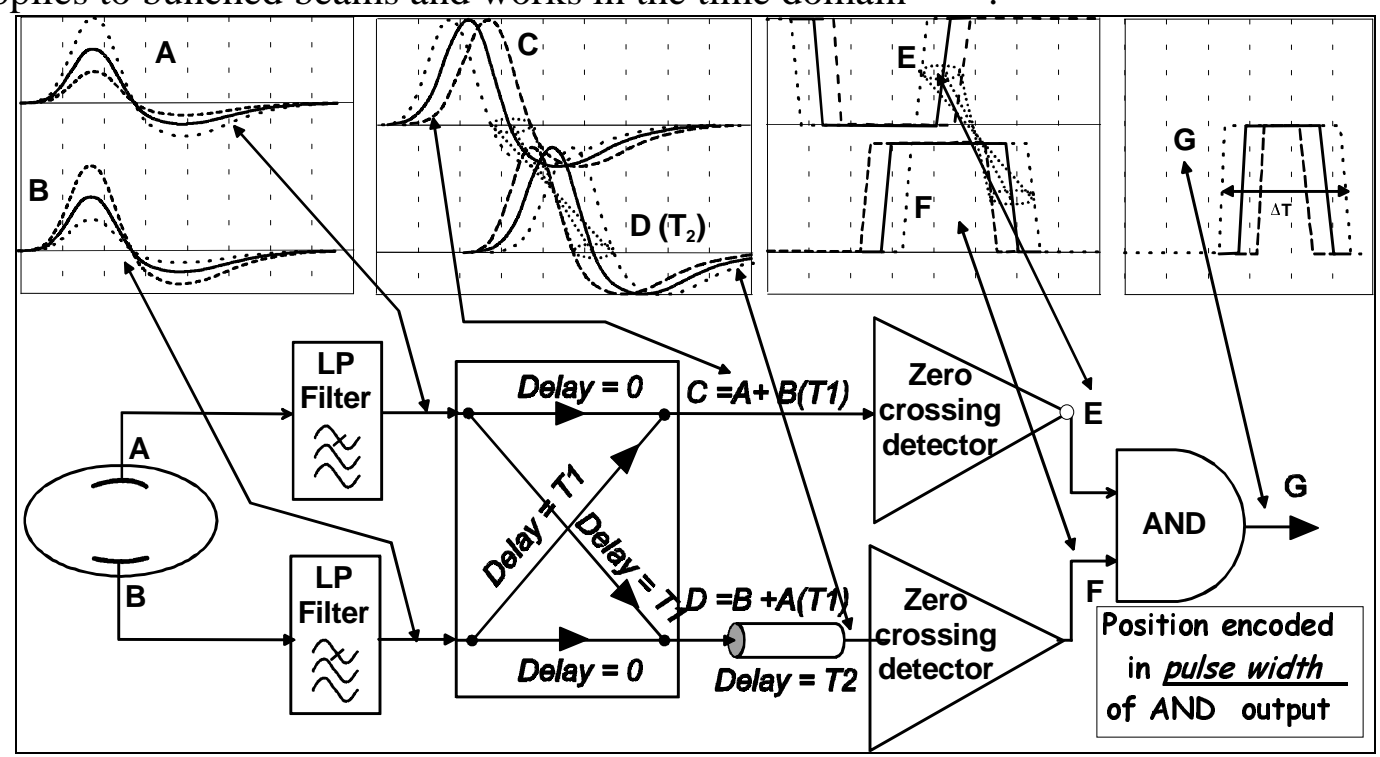

FIGURE 12. Block diagram of the amplitude to time processor 
The LP filters produce the correct pulse shape. The signals from both electrodes are split in two and one branch is delayed by a time $\mathrm{T}_{1}$. The delayed signal of one channel is then added to the direct signal of the other channel, and vice versa. At $\mathrm{C}$, the time of the zero crossing varies according to the signal ratio, up to a maximum of $\mathrm{T}_{1}$. At the output $\mathrm{D}$, you have the same signal amplitude but the time variation has opposite sign.

The maximum time difference is therefore $2 * \mathrm{~T}_{1}$. The delay offset $\mathrm{T}_{2}$ is required to avoid sign ambiguity and should always be larger than $T_{1}$. The zero crossing is independent of amplitude and is easy to detect using fast comparator circuits. Their outputs drive an AND gate, which generates a pulse with a width proportional to the beam position.

$$
\text { Position } \equiv \Delta \mathrm{t}=2 * \mathrm{~T}_{1} *[(\mathrm{~A}-\mathrm{B}) /(\mathrm{A}+\mathrm{B})]+\mathrm{T}_{2}
$$

By integrating this pulse, the time variation is transformed into an amplitude that can be read by an ADC.

TABLE 6. Amplitude to Normalization.

\begin{tabular}{|l|l|}
\hline \multicolumn{1}{|c|}{ Advantages } & \multicolumn{1}{c|}{ Limitations } \\
\hline Fastest normalization process ( $>40 \mathrm{MS} / \mathrm{s})$ & Can only be employed with bunched beams \\
\hline Reduced number of channels (x2) & No Intensity information \\
\hline Input dynamic $>50 \mathrm{~dB}$ & \\
\hline $\begin{array}{l}\sim 10 \mathrm{~dB} \text { reduction on the position dependent } \\
\text { dynamic due to signals recombination }\end{array}$ & Tight time adjustment \\
\hline Dynamic is independent of the number of bunches & Propagation delay between comparators \\
\hline Almost independent of the bunch length & \\
\hline
\end{tabular}

Remark: A specifically designed monolithic Ga-As chip would allow for a large speed breakthrough.

\subsection{Digital Receiver ${ }^{17,18}$}

The digital receiver is a new approach to the classical heterodyne receiver. This has become possible due to technological improvements in the telecommunication field.

In this new approach, the basic functionality is preserved but implementation is different. In fact, as processing and sampling technologies improve, digital treatment moves from the base-band end toward the antenna or the sensor. The present situation allows the digital transition to take place just after the IF amplifier.

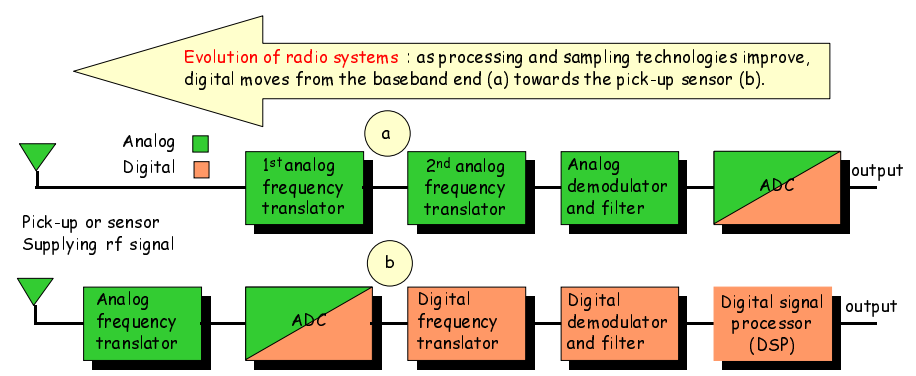

FIGURE 13. Block diagram of the evolution of the heterodyne receivers

The RF front-end circuit, while being identical to its analog counterpart, requires a specific design adapted to the digital world. A BP filter selects the useful signal while rejecting the image frequency and a Pin diode attenuator is used to avoid saturation. 
A frequency down conversion then transposes the signal to the tens of $\mathrm{MHz}$ range $(\sim 36 \mathrm{MHz})$. A surface acoustic wave (SAW) filter with in-band flatness of $\pm 0.1 \mathrm{~dB}$ and a very linear amplifier with adjustable gain to ensure large dynamic range, are required.

The heart of the digital receiver is the analog to digital converter (ADC). Present performances for low cost ADC are: Input bandwidth $(>100 \mathrm{MHz})$; Sampling rate (> $40 \mathrm{MS} / \mathrm{s})$; Resolution (12 bit); Linearity (<.5 LSB). The sampling rate should be chosen to be a few $\mathrm{MHz}$ below the IF frequency in such a way that the signal aliases down to the base band.

Once the IF signal is successfully digitized the next step is the data processing. This could be done by one or more digital signal processor (DSP), which implement the remaining functions (IF filtering, demodulation, data reduction). However, the total processing required in such a receiver may add up to several GFLOPS (giga floating operations per second), which is not a straightforward operation. For this reason specialized digital hardware, known as Digital Down-Converter (DDC) has been designed to perform these specific tasks.

FIGURE 14. Block diagram of a digital down converter

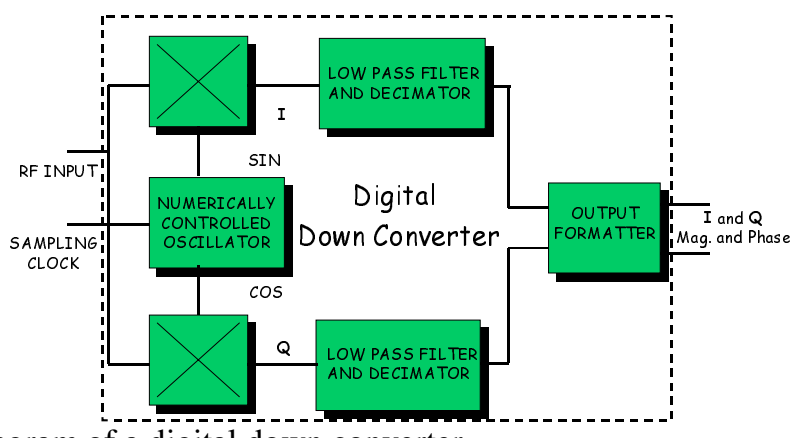

The filtering action defines the processor bandwidth, which in turn affects the system resolution. Since the tuning frequency can easily be re-programmed, the same hardware allows for high speed / medium resolution and low speed high-resolution acquisition.

The data reduction (also called decimation) drastically reduces the downstream signal processing requirements.

The data flow is stored in a FIFO memory and accessed by a DSP that applies the correction factors, does additional filtering, calculates the position and the intensity and performs all other post-processing such as FFTs, etc.

Four identical channels are implemented, one for each electrode in the BPM block.

In order to guarantee an identical and stable gain in each channel, a pilot signal at a frequency shifted by $-1.5 \mathrm{MHz}$ and at a level of -10 to $-20 \mathrm{~dB}$ relative to the beam signal is permanently injected via couplers.

By simply changing the tuning frequency of the digital IF filter, one can choose either the beam or the pilot signals. The digitized pilot signal is used to change the gain in the IF amplifier and keep the global four channel gain within the specified tolerance.

In the classical heterodyne receiver, the most critical parameter is the demodulator non-linearity. When using a digital processor, the demodulation action is done 
digitally by the DDC, hence suppressing this error. Here it is mainly the ADC that defines the global non-linearity.

Another advantage of the digital receiver concerns the resolution. The signal to noise ratio is improved by the digital filtering and the over-sampling with respect to the base band. This factor corresponds to the square root of the ratio of the digital band-pass filter to the Nyquist bandwidth, expressed in $\mathrm{dB}$.

TABLE 7. Digital receiver.

\begin{tabular}{|l|l|}
\hline \multicolumn{1}{|c|}{ Advantages } & \multicolumn{1}{c|}{ Limitations } \\
\hline Programmability & No single shot measurement \\
\hline Narrow \& Wide band processing & No "plug and play" system \\
\hline $\begin{array}{l}\text { Identical gain for all channels due to possible } \\
\text { permanent calibration }\end{array}$ & $\begin{array}{l}\text { All problems related to a new inexperienced } \\
\text { processing system }\end{array}$ \\
\hline Resolution improved by over-sampling techniques & Complex engineering \\
\hline Large dynamic range & \\
\hline Excellent linearity (only limited by the ADC) & \\
\hline
\end{tabular}

\section{APPLICATION EXAMPLE}

The PS and SPS are versatile machines, capable of producing and accepting over 7 different types of beam. The transfer line between the two machines will be equipped with $20 \mathrm{BPMs}$, spread over $1.5 \mathrm{Km}$.

The purpose of this example is to choose the most suitable processing system.

\subsection{Specifications}

\subsubsection{Beam parameters}

TABLE 8. Beam parameters of the 4 most important cases for the PS to SPS transfer line

\begin{tabular}{|c|c|c|c|c|c|c|}
\hline Beam & $\mathbf{N}^{\circ}$ bunches & Spacing & Width & Intensity & Dynamic & Dynamic \\
\hline Fixed target & 2000 & 5 & 1.66 & $1^{* 10^{9}}$ & 30 & 42 \\
\hline LHC & 1 to 84 & 25 & 2.1 & $3^{* 10^{9}}$ & 35 & 86 \\
\hline Special beam & $1,8,16$ & 131.2 & 4.8 & $5^{*} 10^{9}$ & 20 & 56 \\
\hline Heavy ions & 16 & 131.2 & $6.2 / 15$ & $2.6^{*} 10^{8}$ & 10 & 22 \\
\hline & $\mathrm{N}$ & $\mathrm{ns}$ & $(\mathbf{f w h m}) \mathrm{ns}$ & Charge/bunch & (Bunch) dB & (Global) dB \\
\hline
\end{tabular}

Analysis of the beam parameters (Table 8) reveals the following difficulties:

- A very wide dynamic range $(>86 \mathrm{~dB})$, which comprises bunch intensity variation, variable number of bunches and $12 \mathrm{~dB}$ of position dynamic range.

- A variable bunch width, of an order of magnitude.

- Bunch spacing from 5 ns to a single bunch. 


\subsubsection{Processing system specifications}

The required accuracy and linearity should be contained in $2 \%$ of the $\mathrm{N}_{\mathrm{a}}$ for a nominal beam, while resolution and stability versus intensity should be $<0.5 \%$ of the $\mathrm{N}_{\mathrm{a}}$.

The PS extraction time is such that beam duty cycle is very low in the transfer line hence limiting the integrated radiation to an acceptable value.

Considering the long distances between the PUs and the electronics racks and taking into account the high frequency signal spectrum, it is advisable to place the processing electronics as near as possible to the PUs.

\subsection{Pick-up choice}

For a beam intensity of a few $10^{8} \mathrm{p} / \mathrm{b}$, the choice of a coupler offers a better sensitivity compared to buttons. The coupler length should be optimized to obtain a maximum response in the frequency range from a few $\mathrm{MHz}$ up to $200 \mathrm{MHz}$. This corresponds to a coupler length of $50 \mathrm{~cm}$, with an azimuthal coverage of $24^{\circ}$ and $\mathrm{Z}_{\mathrm{t}}=$ $7 \Omega$

\subsection{Spectral analysis}

The variable frequency signal spectral content makes it difficult to choose whether a wide or narrow band processor is most suited to this application.

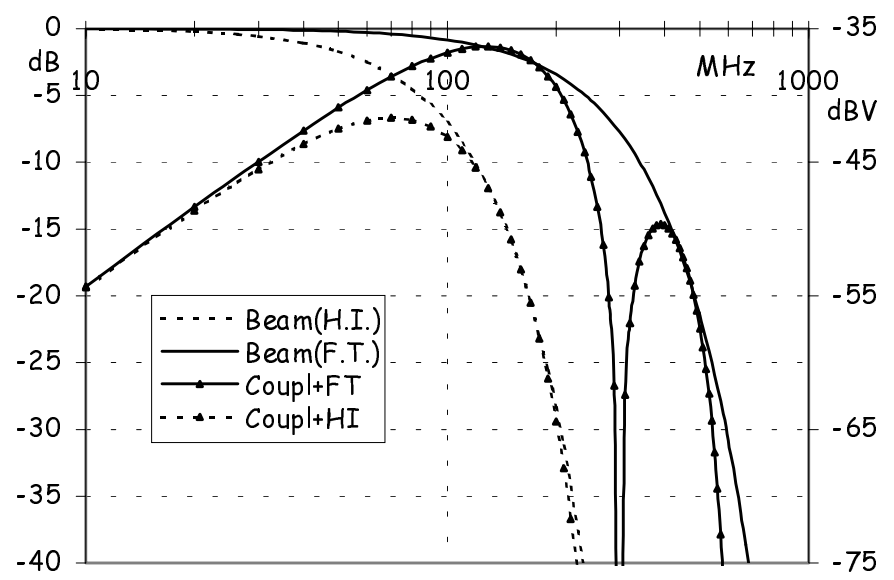

FIGURE 15. Beam and coupler signal frequency spectrum.

A "Spice" simulation of 3 particular cases (fixed target, LHC and single bucket filling) will help for a better understanding.

Let's take a wide-band system with a low-pass frequency of $200 \mathrm{MHz}$ and a band-pass filter centered at $200 \mathrm{MHz}$, having a $12 \mathrm{MHz}$ bandwidth. For a real narrow-band system $(\approx 1 \mathrm{MHz})$ you can linearly scale down the results of the above-simulated BP filter.

Figures 16, 17 and 18 show the frequency contents and the time domain response. 

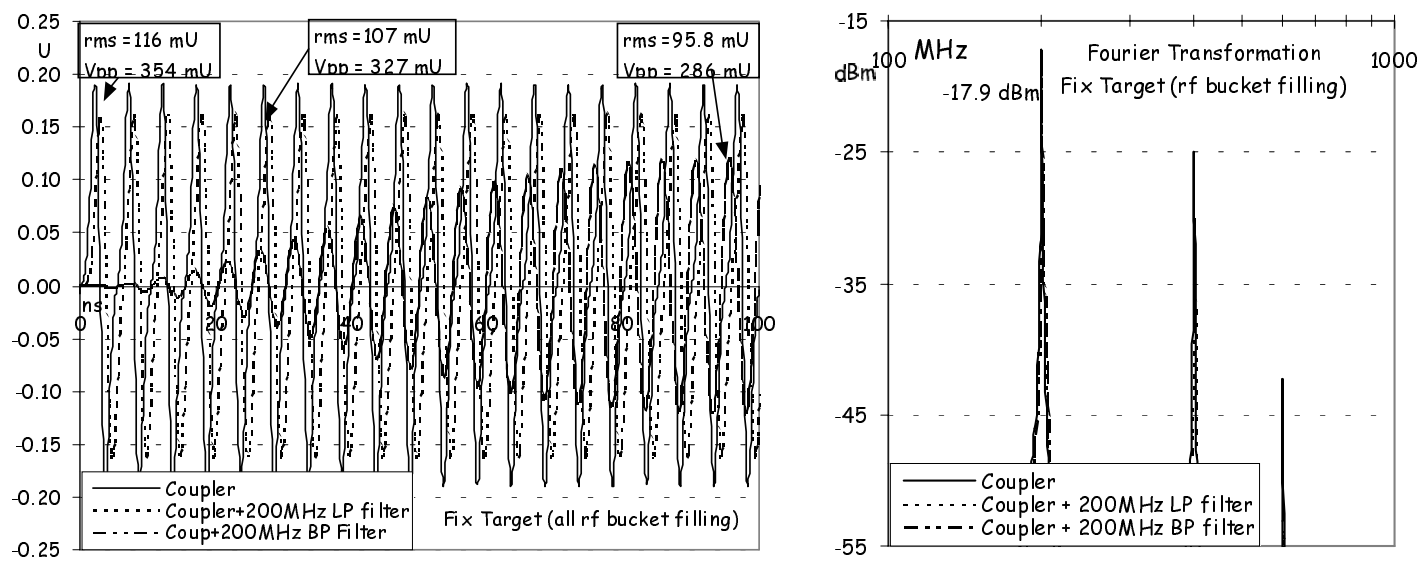

FIGURE 16. Fixed target (all $\mathrm{rf}$ buckets filled). Time response and spectral content
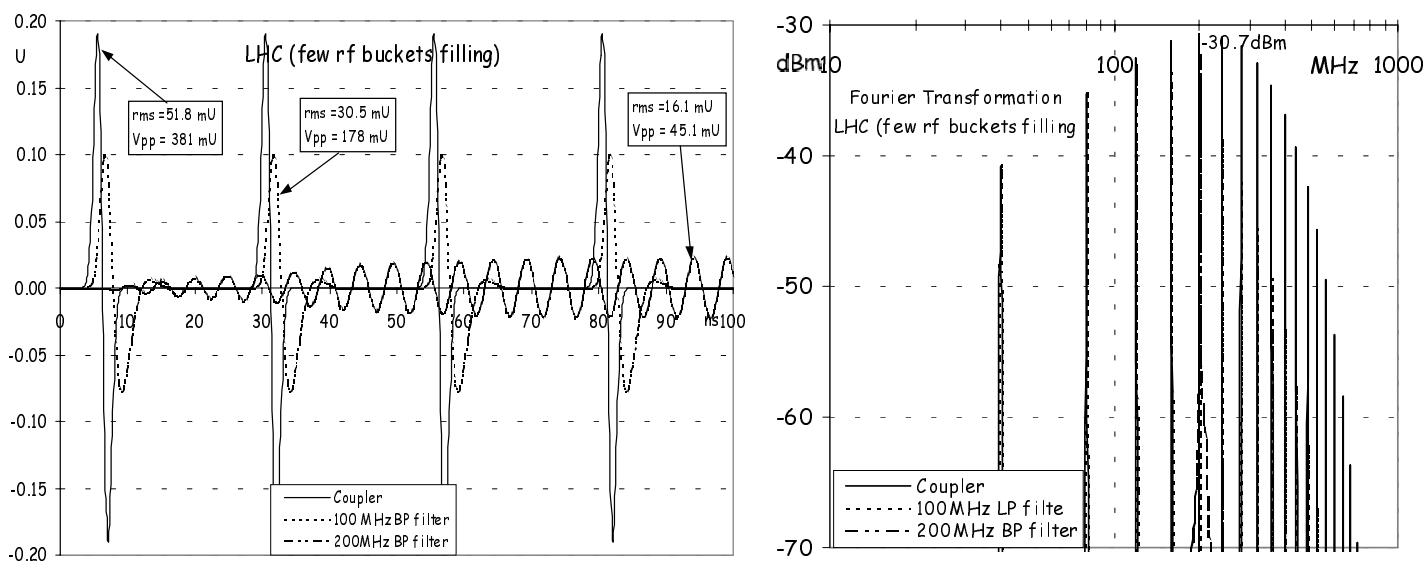

FIGURE 17. LHC (few rf buckets filled). Time response and spectral content
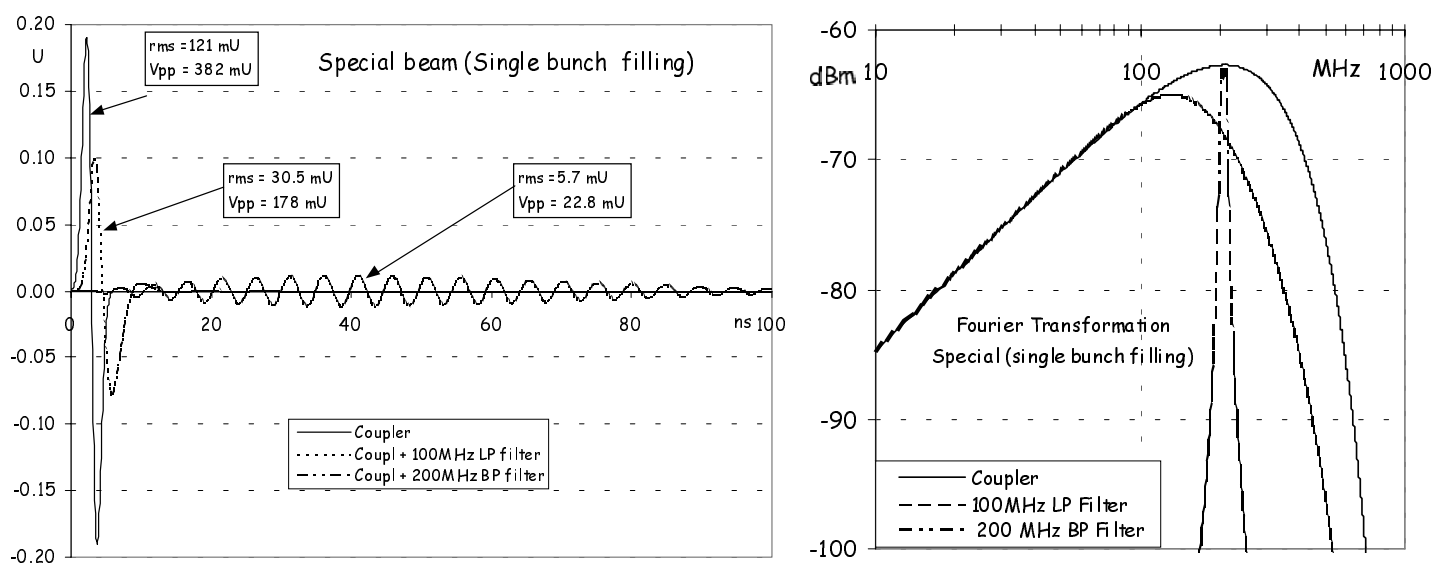

FIGURE 18. Single bunch. Time response and spectral content

\subsection{Spectral content and time response.}

Assuming an identical global input noise of $1.6 \mu \mathrm{V} / \sqrt{\mathrm{MHz}}$, the $\mathrm{S} / \mathrm{N}$ ratio is indicated in the following table. 
TABLE 9. S/N considerations

\begin{tabular}{|c|c|c|c|}
\hline Processor & Wide-band & Narrow-band & \\
\hline Band-width & 200 & $12.0 \quad(1.2)$ & $\mathrm{MHz}$ \\
\hline Input noise & 23.0 & $5.4 \quad(1.7)$ & $\mu \mathrm{V}$ (rms) \\
\hline & $\mathbf{S} / \mathbf{N}$ & $\mathbf{S} / \mathbf{N}$ & \\
\hline Fixed Target & $\begin{array}{ll}76.8 & {[89.8]} \\
\end{array}$ & $86.4(96.4)$ & $\mathrm{dB}$ \\
\hline LHC & $\begin{array}{ll}75.8 & {[82.6]} \\
\end{array}$ & $70.2(80.2)$ & $\mathrm{dB}$ \\
\hline Single $(4.8 n s)$ & $67.1 \quad[67.1]$ & $39.8(29.8)$ & $\mathrm{dB}$ \\
\hline
\end{tabular}

Values in [] correspond to the possible improvement one can obtain when averaging the collected data in a time interval equivalent to that required by a NB.

It should be noted that NB processing performs better for a limited frequency spectrum (Fixed Target) but as soon as the frequency spectrum becomes larger, WB processing becomes superior.

In the case of a single bunch, NB performance drops dramatically with the bunch length. The reason is that the first part of the bunch excites the filter but after half an oscillation period, the filter polarity changes and the input signal starts annihilating what has already been filled.

\subsection{Processing system choice}

TABLE 10. Processing system comparison

\begin{tabular}{|c|c|c|}
\hline Processor type & Remarks & Solution $=$ \\
\hline MPX heterodyne & MPX, PLL and AGC don't work for single passage. & Unfeasible \\
\hline NB $\Delta / \Sigma$ & $\begin{array}{l}200 \mathrm{MHz} \text { tune, cannot detect Heavy Ions at } 7.6 \text { or one of its } \\
\text { harmonics. }\end{array}$ & Unfeasible \\
\hline Phase Normalizer & $\begin{array}{l}200 \mathrm{MHz} \text { discriminator \& logic is difficult to realize. } \\
\text { Insufficient sensitivity for weak and long bunches. }\end{array}$ & Unfeasible \\
\hline Time Normalizer & $\begin{array}{l}\text { Not suitable for variable bunch widths. Electronics time jitter } \\
\text { is too big relative to the possible time excursions. }\end{array}$ & Unfeasible \\
\hline WB $\Delta / \Sigma$ & $\begin{array}{l}\text { Requires several gain switching and scaling factors. } \\
\text { Difficult to calibrate. }\end{array}$ & Feasible \\
\hline Digital receiver & $\begin{array}{l}\text { Easy switching from } 200 \text { down to } 198.1 \mathrm{MHz} \text { tuning } \\
\text { frequency. Adjustable BW. No AGC action, hence larger } \mathrm{N}^{\circ} \\
\text { of bits }\end{array}$ & Feasible \\
\hline Log-amplifier & $\begin{array}{l}\text { Excellent dynamic range. Insensitive to bunch shape \& length. } \\
\text { Reduced } \mathrm{N}^{\circ} \text { of bits. Low cost. Limited linearity }\end{array}$ & Feasible \\
\hline
\end{tabular}

The above table collects the main advantages / disadvantages of the various processing systems. Three solutions appear to be feasible but just one offers almost no restrictions.

Since resolution and accuracy are not restrictive parameters for this application, the logarithmic processor is by far superior to all the others systems. 


\section{CONCLUSIONS}

Experience has proved that every new machine requires a new approach to the same problem. This offers an excellent opportunity for new ideas.

Several different combinations among the currently available processing systems have still to be explored and this may provide solutions for particular cases.

The telecommunications field has similar needs and is expanding rapidly. Full advantage should be taken of its technological progress and of the reduced prices of components on the consumer market.

My personal feeling for the future is that two processing systems, capable of covering almost all possible applications, will coexist: the Amplitude to Time Normalizer and the Digital Receiver. Both have a bright future.

\section{ACKNOWLEDGMENTS}

I would like to thank Dr. Hermann Schmickler for his encouragement and precious advice for structuring this subject and my most faithful collaborator J.L. Pasquet who has built and tested most of the circuits described here.

\section{REFERENCES}

1 A. Hofmann, "Beam diagnostics and applications", BIW 98, pg.3.

2 F. Loyer, K. Scheidt, "Electron beam position monitor : performances considerations", Dipac 93 Proc, pg. 21.

3 J. Matheson "Non-linearity in the response of BPM", same proceeding

4 R. Lorentz, "Cavity beam position monitor", BIW 1998 Stanford CA, AIP Conf Proc. 451, pg 74

5 R. Biscardi, J.W.Bitter, "Switched detector for Beam Position Monitor", Proc. IEEE Particle Accelerator Conference, San Francisco CA (1991) Cat N89CH2669-0, Vol.3, pp.1154 - 1156.

6 J.A.Hinkson, K.B. Unser, "Precision analog signal processor for BPM in electron storage ring", Dipac 95 Proc, Travemunde (D).

7 M. Le Gras , D.J. Williams, "Trajectory measurement of bunches ejected from the CERN PSB”, Dipac 97 Proc., pg.

8 R. Bossart et al. "Synchronous receivers for beam position measurement", IEEE trans. Nucl. Sci. 32, 1899 (1985).

9 R.E. Shafer, G. Swain et ali. "Beam position monitor upgrade for Los Alamos proton storage ring", U.S.PAC Conf. Proc.(1989), page 1562.

10 G.Aiello, M.R.Mills, "Log-ratio technique for beam position monitor systems", BIW92, AIP Conf. Proc. xxx, page 301 .

11 R.E. Shafer, "Log-ratio signal processing technique for beam position monitors", BIW92, AIP Conf. Proc. xxx, page 120.

12 G. Vismara SL/BI Note 1998.

13 F.D. Wells, S.P. Jachim, "A technique for improving the accuracy and dynamic range of BPM", PAC Proc. 1989.

$14 \mathrm{~J}$. Borer et al. "The second generation and optimized beam orbit measurement system of LEP. Hardware and performance description", $2^{\text {nd }}$ DIPAC, Travemunde 1995.

15 G. Vismara et al. "From narrow to wide band normalizer for orbit and trajectory measurements", BIW 98, AIP conference proceeding 451, page 246.

16 D. Cocq " The wide band normalizer - a new circuit to measure transverse bunch position in accelerator and collider", NIM A 416 (1998) I-8.

17 R. Ursic et al. "Digital receiver offer new solutions for beam instrumentation", PAC 1999 Proc., pg. 2253

18 M. Dehler, et al. "BPM system for the swiss light source”, Proceedings 1999 PAC, pg. 2087 